\title{
DUST FROM THE COMETS
}

\author{
Tadashi Mukai \\ Kanazawa Institute of Technology \\ Nonoichi, Ishikawa 921, Japan
}

\begin{abstract}
We have found a similarity between the size spectra of the observed interplanetary dust and the survived cometary dust, referring to the dynamical behaviour of the dust leaving the comet. As a result, we can suggest that short-period comets with relatively higher eccentricities are major source of the interplanetary dust, especially those with radii less than $10 \mu \mathrm{m}$. It is predicted, furthermore, that a supply rate of the dust, which move on bound orbits after leaving the comet, becomes about $8 \times 10 \mathrm{~g} / \mathrm{s}$ from 85 short-period comets, and nearly $3 \times 10^{4} \mathrm{~g} / \mathrm{s}$ from $101 \mathrm{long}$-period comets.
\end{abstract}

\section{INTRODUCTION}

One of the important questions for the interplanetary dust is where they come from. It is well known that the comets supply the dust in the solar system. Recently, Comet Halley flybys have brought a lot of valuable information of the dust in the comet, such as the mass spectrum of the cometary grains (e.g. McDonnell et al. 1987, and Mazets et al. 1987) and their chemical composition(e.g. Jessberger et al. 1986).

The data of the dust near the earth's orbit are also surely increasing. The analysis of the particles collected in the upper atmosphere of the earth provides us a powerfull tool to examine their origin and evolution in the interplanetary space. The size spectrum of the interplanetary dust, compiled based on the research of the lunar microcraters plus in situ measurements of dust flux, is also available to investigate their origin.

In this paper, I will study the interrelation between the dust in the comet and those in the interplanetary space based on a comparison of their size spectra. Dynamical evolution of the dust ejected from the comet is mainly considered to estimate the size spectrum of the survived cometary grains against an emission velocity of the grain from the cometary nucleus as well as the solar radiation pressures. As a result, it becomes possible to re-attack the old problem, i.e. how much dust can be supplied from the comets into the solar system. 


\section{SIZE SPECTRUM OF THE DUST}

By using a variable mass density of the dust, decreasing with increasing radius, predicted in Lamy et al. (1987), the mass spectrum of the dust in comet Halley compiled by Divine and Newburn(1987) is converted to the size spectrum of the dust in the comet(see figure la). The data of the size spectrum derived from three different in situ data have shown slightly different features, but the existence of the smaller grains with radii $s<0.1 \mu \mathrm{m}$ has been proved.

On the other hand, figure $1 \mathrm{~b}$ shows the size spectrum of the dust near the earth's orbit compiled by Grün et al.(1985)(GZFG in figure) and Le Sergeant and Lamy(1980)(LL in figure). Due to the difference of calibration of the microcrater analysis, the slopes of the grains with $s<1 \mu \mathrm{m}$ in both estimations are in disagreement.

From a comparison between figures $1 \mathrm{a}$ and $1 \mathrm{~b}$, we have a feeling that the dust with radii between $0.01 \mu \mathrm{m}$ and $10 \mu \mathrm{m}$ decreases during their dispersion from the comet to the interplanetary space. This is the initial motivation of the following study.

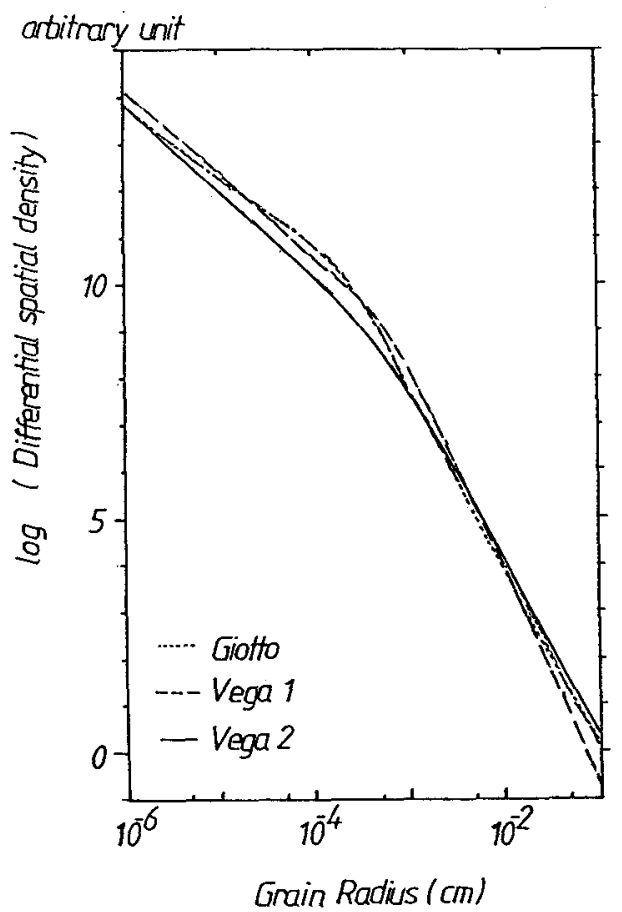

Fig. 1a. Size spectrum of the dust detected in comet Halley.

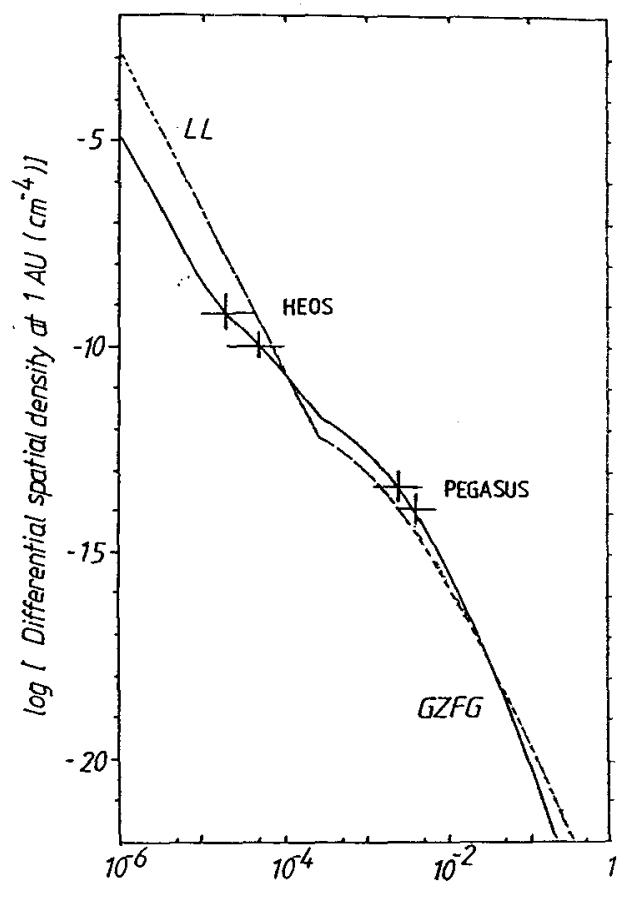

Grain radius $(\mathrm{cm})$

Fig.1b. Size spectrum of the dust near the earth's orbit. GZFG is Grïn et al. (1985) and LL denotes LeSergeant and Lamy(1980). 


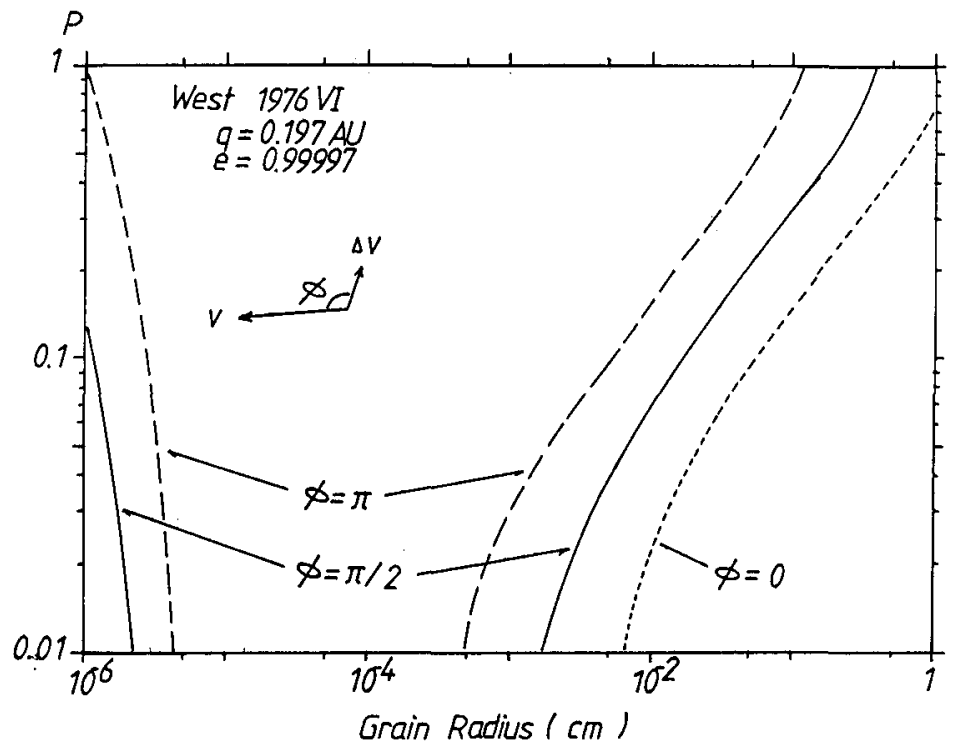

Fig.2a. Survival probability $P$ of the dust ejected from long-period comet during its one orbit period. $v$ denotes an orbital velocity of the parent comet and $\Delta \mathrm{v}$ means an emission velocity of the particle from the comet.

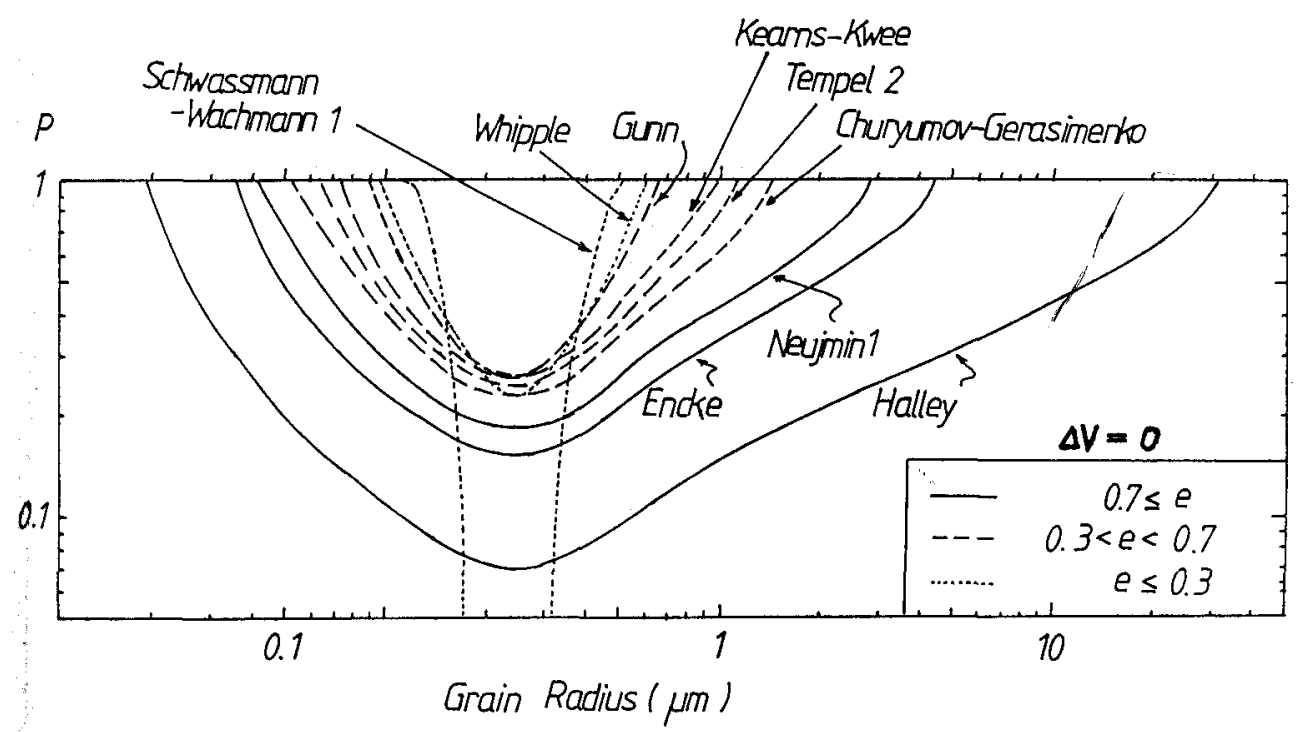

Fig.2b. The same as fig.2a, but those from short-period comets. 


\section{SIZE SPECTRUM OF SURVIVED COMETARY DUST}

It is known that a part of the ejected grains cannot stay in the solar system after leaving the comet, because their orbital energy becomes positive due to the solar radiation pressures on them and their emission velocities $\Delta v$ from the comet. Mukai(1985) has defined the survival probability $P$ of the dust leaving the comet during one perihelion passage of the parent comet. Figure 2 shows the results of $P$, as a function of a grain radius $s$, computed for one long-period comet (comet west 1976VI) and 6 short-period comets. For a ratio of the solar radiation pressure to the solar gravity on the dust, the results for silicate given in Mukai(1985) were applied. In addition, the values of $\Delta v$ came from an empirical formula in Mukai et al. (1985), estimated for comet Halley as functions of the grain radius and the sun-comet distance. These values of $\Delta v$, decreasing with increasing the grain radius, are roughly two order of magnitude smaller than the orbital velocity of the parent comet.

Figure 2 tells us that long-period comet scarcely provide the grains with radi $i$ less than $10 \mu \mathrm{m}$ even in the case of backward ejection. Furthermore, it is found that for short-period comets, the depression in the curve of $P$ near $s=1 \mu \mathrm{m}$ becomes wider and deeper as the eccentricity of the parent comet increases, and finally it would approach the feature found in long-period comet. If one considers only a perihelion ejection of the grain from the comet, no survived grains with radi smaller than some critical radius sc appear. The value of sc increases with increasing the sun-comet distance r. Since the allowable range of $r$ for the comet with nearly circular orbit is very limited, then the depression in $P$ becomes sharp and deep, as shown in figure 2 .

We assume that the size spectrum of the cometary dust is independent of $r$. Therefore, the size distribution of survived cometary dust is estimated from a multiplication of $P$ derived above by the size spectrum of cometary dust (Vega 2 data shown in figure 1a)

Fig.3a. Resulting size spectrum of survived dust ejected from short-period comets.

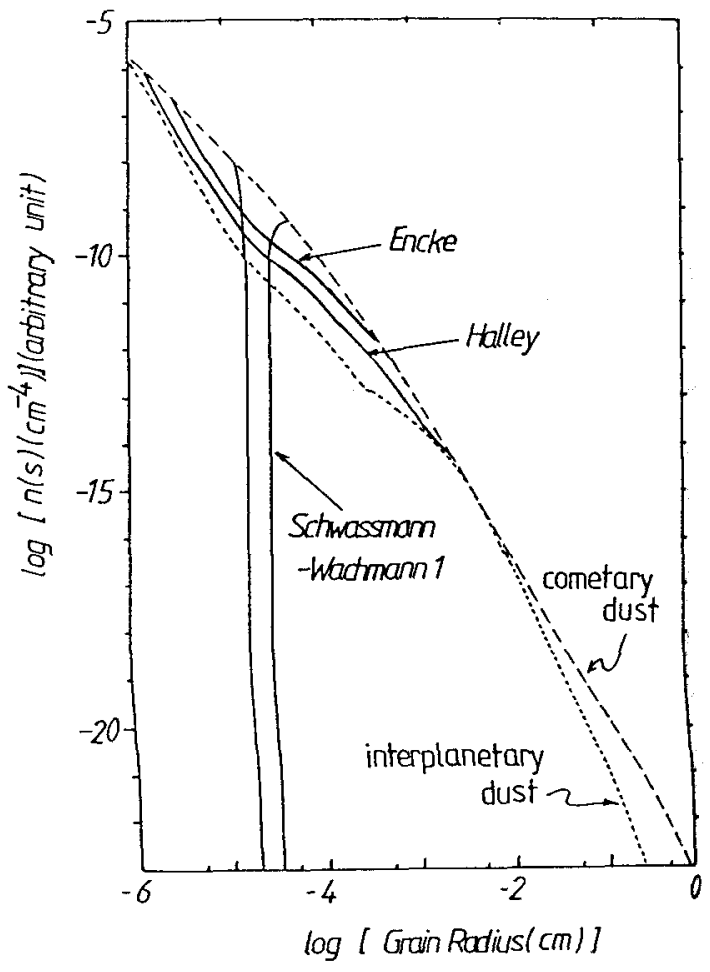


It is found that the resulting size spectrum of survived cometary dust ejected from, especially, short-period comets with larger eccentricities has a similar depression of the grains with radii between $0.01 \mu \mathrm{m}$ and 10 $\mu \mathrm{m}$ to that found in the interplanetary dust(GZFG in figure $1 \mathrm{~b}$ ) (see figure 3 ).

on the contrary, the shape of the size spectrum expected for survived grains supplied from long-period comets and from short-period comets with nearly circular orbits is quite different, compared with that observed in the interplanetary dust. Since most of the asteroids have the nearly circular orbits, the survived dust released from the asteroids would have also sharp and deep depression feature in $\mathbf{s}<10 \mu \mathrm{m}$, although the process of dust ejection in the asteroids is unlike that in the comets. of course, we cannot conclude from this result alone that all of the interplanetary dust come from short-period comets with relatively higher eccentricities. It might be problem to assume that all comets have the same size spectrum of the dust as that observed in camet Halley. Furthermore, a modification of the size spectrum of the dust in the interplanetary space, such as a production of smaller debris by mutual collisions of the interplanetary dust, should be taken into account in the future study. However, the similarity of the shape of the depression of the grains with radii between $0.01 \mu \mathrm{m}$ and 10 $\mu \mathrm{m}$ discussed above strongly suggests that short-period comets play an important role for the supplier of the interplanetary dust, at least, those with radii less that $10 \mu \mathrm{m}$.

\section{HOW MUCH DUST COME FROM THE COMETS?}

A large fraction of the mass supplied from the comets is in the grains with radii larger than $100 \mu \mathrm{m}$. Therefore, the depression of the grains with radii less than $10 \mu$ predicted above becomes out of the consideration when the total mass supplied from short-period comets is 


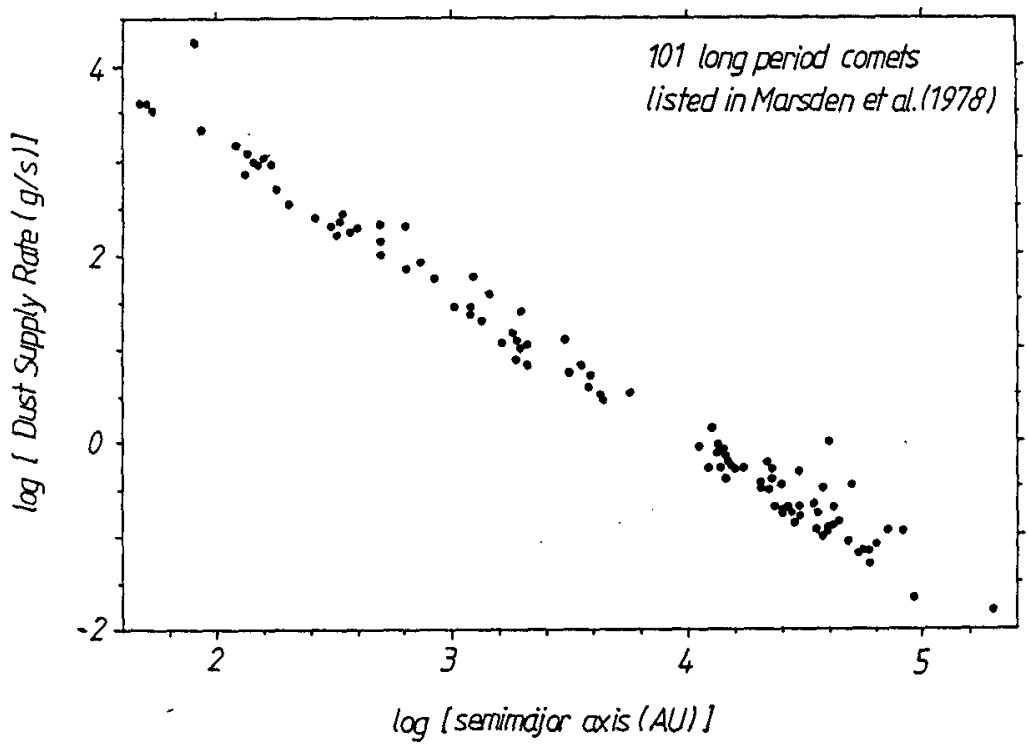

Fig. 4a. Mass supply rate of the dust from long-period comets.

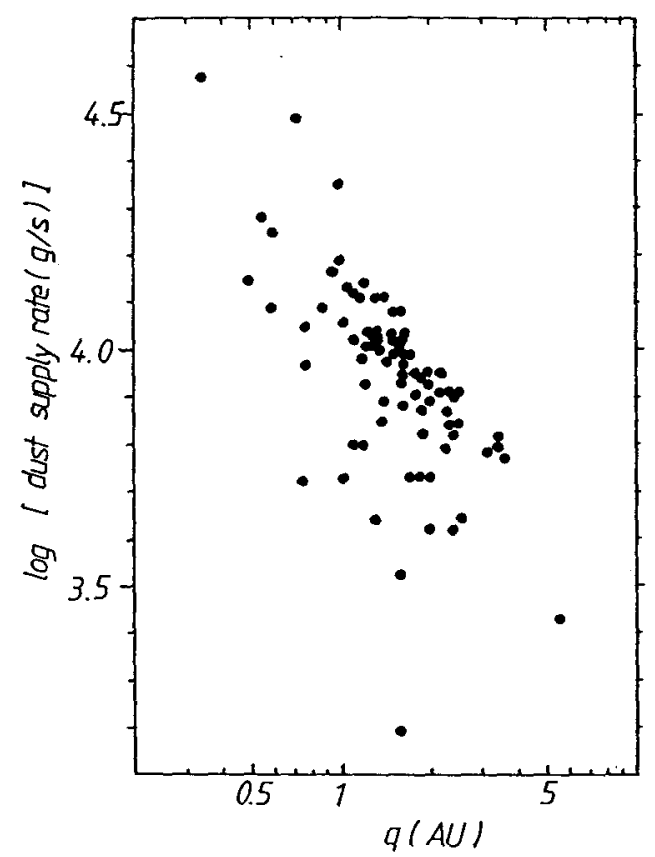

Fig. 4b. The same as fig.4a, but from 85 short-period comets, 
examined. On the other hand, for the mass supplied from long-period comets, such depression plays a key factor.

The dust production rate of the comets is assumed to be approximated as $10^{5} \mathrm{r}^{-2} \mathrm{~g} / \mathrm{s}$ for short-period comets and $10^{6} \mathrm{r}^{-2} \mathrm{~g} / \mathrm{s}$ for long-period comets, where $r$ is a sun-comet distance in unit of $A U$ (see Ney 1982). After that, the total mass of dust supplied from the comets are computed(see figure 4). The long-period comet with larger semimajor axis can supply less amount of the dust because the survival probability $P$ for such a comet is, in general, low in the wide range of the grain radius. On the other hand, mass supply rate from shortperiod comet increases as the perihelion distance $q$ of the comet decreases. This result is easily understood since the comet with smaller value of q can produce much dust near the sun.

Adding these computed values of the mass supply rates from 85 short-period comets and 101 long-period comets(the orbital data, respectively, came from Marsden 1986 and Marsden et al. 1978 ), we can predict that the total mass of dust supplied from short-period comets becomes $8 \times 10^{5} \mathrm{~g} / \mathrm{s}$, and that from long-period comets is $3 \times 10^{4} \mathrm{~g} / \mathrm{s}$. These results seem to be consistent with the values previously estimated by other authors based on the different way, e.g. Röser(1976) derived $2.5 \times 10^{5} \mathrm{~g} / \mathrm{s}$ from short-period comets.

The uncertainties in the above estimation arise from the data of the dust production rate of the comets. The observed dust production rates scatter within the scale of one order of magnitude from comet to comet even at the same sun-comet distance r(see e.g. Hanner 1984). The dependence of dust production rate on $r$ should be examined in detail in the future works.

\section{Conclusion}

Referring to the similarity of the shape of depression in the size spectrum of the observed interplanetary dust and that of the survived dust released from short-period comets, the origin of the interplanetary dust was discussed. It is found that short-period comets with high eccentricities play an important role for dust supplier, especially those with $\mathrm{s}<10 \mu \mathrm{m}$. In addition, the contribution of the mass of dust supplied from long-period comets is roughly 30 times smaller than that from short-period comets.

Recently, IRAS found the dust bands associated with the orbits of the asteroids. As discussed above, the size spectrum of the survived dust released from the asteroids is supposed to have a different shape in the region of the grain radius less than $10 \mu \mathrm{m}$, compared with the observed shape of the size spectrum of the interplanetary dust. However, the thermal emission detected by IRAS mainly came from the grains larger than $s=10 \mu \mathrm{m}$. This implies that the contribution of the dust with larger radii from the asteroids may be important in the interplanetary space. Further quantitative discussions about the dust supplied from the asteroids, such as the size spectrum of the survived asteroidal dust and the total mass of dust supplied from the asteroids, are strongly needed. 


\section{ACKNOWLEDGEMENT}

This work was partially supported by the Grant-in-Aid for Scientific Research on Priority Areas(Origin of the Solar System) of Japanese Ministry of Education, Science, and Culture(No.63611007).

\section{REFERENCES}

Divine,N., and Newburn,RL,Jr.(1987). Modeling P/Halley before and after the encounters. Astron. Astrophys, 187, 867-872.

Grün,E., Zook,H.A., Fechtig,H., and Giese,R.H. (1985). Collisional balance of the meteoritic complex. Icarus, 62, 244-272.

Hanner,M.S. (1984). A comparison of the dust properties in recent periodic comets. Adv. Space Res. 4, 189-196.

Jessberger,E.K., Kissel,J., Fechtig,H., and Krueger,F.R.(1986). On the average chemical composition of cometary dust. ESA EP-249, 27-30.

Lamy, P.L., Grün,E., and Perrin,J.M. (1987), Comet P/Halley:implication of the mass distribution function for the photopolarimetric properties of the dust coma. Astron. Astrophys. 187, 767-773.

LeSergeant d'Hendrecourt,L.B., and Lamy,P.L.(1980). On the size distribution and physical properties of interplanetary dust grains. I carus, $43,350-372$.

Marsden,B.G. (1986). Catalogue of Cometary Orbits(5th ed.)(Smithsonian As trophysical Observatory, Cambridge, MA).

Marsden,B.G., Sekanina,Z., and Everhart,E. (1978). New osculating orbits for 101 comets and analysis of original orbits for 200 comets. Astron. J., 83, 64-71.

Mazets,E.P., Sagdeev,R.Z., Aptekar,R.L., Golenetski i,S.V., Guryan,Yu.A. , Dyachkov,A.V., Ilyinski i,V.N., Panov,V.N., Petrov,G.G., Savvin,A.V., Sokolov, I.A., Frederiks,D.D., Khavenson, N.G., Shapiro,V.D., and Shevchenko,V.I.(1987). Dust in comet P/Halley from Vega observations. Astron. Astrophys. 187, 699-706.

McDonnell,J.A.M., Alexander,W.M., Burton,W.M., Bussoletti,E., Evans, G.C., Evans,S.T., Firth,J.G., Grard,R.J.L., Green,S.F., Grün,E., Hanner,M.S., Hughes, D.W., Igenberg, E., Kissel, J., Kuczera,H., Lindblad,B.A., Langevin,Y., Mandeville, J.-C., Nappo,S., Pankiewicz,G.S.A., Perry,C.H., Schwehm,G.H., Sekanina,Z., Stevenson,T.J., Turner,R.F., Weishaupt, V., Wallis,M.K., and Zarnecki,J.C.(1987). The dust distribution within the inner coma of comet P/Halley 1982i:encounter by Giotto's impact detectors. Astron. Astrophys. 187, 719-741.

Mukai,T. (1985). Small grains from comets. Astron. Astrophys. 153, 213-217.

Mukai,T., Mukai,S., Fechtig, H., Grün,E., and Giese,R.H.(1985). Adv. Space Res. 5, 339-342.

Ney,E.P.(1982). Optical and infrared observations of bright comets in the range of $0.5 \mathrm{um}$ to $20 \mathrm{um}$. In Comets(L.L.Wilkening, Ed.). pp. 323-340, Univ. of Arizona Press, Tucson.

Röser,S.(1976). Can short period comets maintain the zodiacal cloud? Lecture Notes in Physics 48, 319-322. 